\title{
Mosaic supernumerary ring chromosome 19 identified by comparative genomic hybridisation
}

\author{
Saeed R Ghaffari, Elizabeth Boyd, J Michael Connor, Alison M Jones, J L Tolmie
}

\begin{abstract}
We report the use of comparative genomic hybridisation (CGH) to define the origin of a supernumerary ring chromosome which conventional cytogenetic banding and fluorescence in situ hybridisation (FISH) methods had failed to identify. Targeted FISH using whole chromosome 19 library arm and site specific probes then confirmed the CGH results. This study shows the feasibility of using CGH for the identification of supernumerary marker chromosomes, even in fewer than $50 \%$ of cells, where no clinical or cytogenetic clues are present.

( Med Genet 1998;35:836-840)
\end{abstract}

Keywords: chromosome 19; comparative genomic hybridisation; ring chromosome; mosaicism

The origin of supernumerary marker chromosomes cannot always be resolved by means of conventional banding techniques. ${ }^{1}$ When there are cytogenetic or clinical clues to the chromosome abnormality, FISH using appropriate probes may identify the origin of the marker chromosome, otherwise a stepwise approach using different FISH probes or reverse chromosome painting, ${ }^{2-5}$ possibly combined with chromosome microdissection, is required. ${ }^{6-10}$ However, when the marker chromosome is very small, or has no significant band landmark, or is present in a low percentage of cells only, even FISH may fail to identify the abnormality. Both FISH and microdissection also depend on good quality metaphases with high mitotic index, conditions that may be difficult to obtain. Therefore, a genome wide screening technique is desirable.

Comparative genomic hybridisation (CGH)

Institute of Medical Genetics, Yorkhill Hospitals Campus, Yorkhill, Glasgow G3 8SJ, UK $S$ R Ghaffari E Boyd

J M Connor

J L Tolmie

Merchiston Hospital, Brookfield, By Johnstone PA5 8TY, UK

A M Jones

Correspondence to: Dr Boyd.

Received 13 November 1997 Revised version accepted for publication 13 March 1998 is a genome wide screening technique that was first developed to study chromosome abnormalities in tumours. ${ }^{11-13}$ It has also been used as an adjunct to conventional cytogenetics for the diagnosis of chromosome imbalance in clinical cytogenetics. ${ }^{14-16}$ However, there are no reports of CGH being used as a tool to diagnose the origin of the extra DNA material in patients who have low percentage mosaicism for a small, supernumerary, marker chromosome.

Here we report a 71 year old woman with mental retardation and a supernumerary marker chromosome, the genetic origin of which was not detected despite attempts using conventional cytogenetics and, latterly, FISH using centromeric probes for a number of chromosomes. In contrast, $\mathrm{CGH}$ readily showed its origin from chromosome 19 and

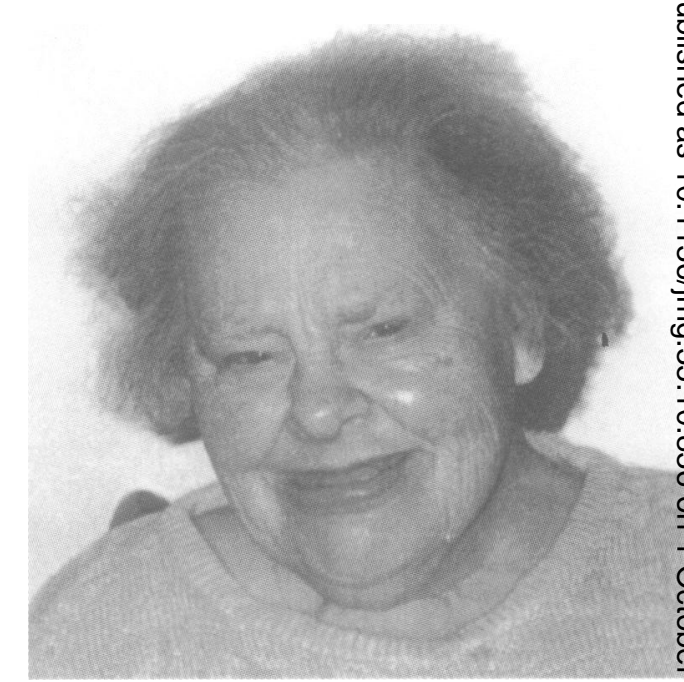

Figure 1 The proband.

complementary experiments by FISH con firmed the $\mathrm{CGH}$ results.

\section{Materials and methods \\ CASE REPORT}

The proband (fig 1), a 71 year old woman with mental handicap, had been in residential car? for 55 years. No details of her birth history of antenatal life were available. The famil recalled her walking at the age of 2 years and. the development of speech was delayed unt the age of 4 or 5 years. She was not clumsy bu㑋 always had some difficulty with fine motor. skills. She attended a private convent school is early childhood but upon her mother's death? when the proband was aged 16 years, she was received into care and has resided in institu tions ever since. The proband was the nint child born in a family of 10 . In the family, nfo sib was similarly affected to the proband and there was no history of mental or physicas illness. In 1976 the proband was admitted to hospital for treatment for hypertension and impaired renal function of unknown origin. At that time, she also had septic skin lesions an osteoarthritis affecting the hips. At present, sheg is able to feed, wash, and dress herself (with) some assistance), handle toys, obey simple commands, and guard against physical dan $\vec{\oplus}$ gers. Speech is mostly indistinct but she cap utter short simple sentences that convey mean ing. She is able to write a few words and caf also read several words, but is notably skilled at. completing jigsaw puzzles. It is thought oste $\overline{-}$ arthritis has led to loss of some fine motor skills. The proband has never been independent or able to go out on her own but she enjoys social events. She has urinary and bowel continence. 
Physical examination showed a height of 132 $\mathrm{cm}$, head circumference of $52.5 \mathrm{~cm}$, blood pressure of $180 / 110 \mathrm{~mm} \mathrm{Hg}$, poor peripheral circulation, early hypertensive changes in both optic fundi, poor vision, upward slanting palpebral fissures, large tongue, protruding lower lip, normal palmar creases, normal female genitalia, and deep set toe nails. She has reduced muscle power, a shuffling gait, bilateral pes planus, and radiological signs of subluxed, osteoarthritic hip joints. An $x$ ray in 1994 was reported to show considerable degenerative changes in the cervical spine with gross osteophyte formation and partial, presumed congenital, fusion of vertebral bodies C3 and C4. Recently, renal function has declined with recurrent urinary infections and her right kidney is non-functioning.

Detailed psychological examinations were performed and the highest estimate of intellect suggested moderate to mild learning disability. However, the British Picture Vocabulary Scale result and the examiners' impressions indicated that moderate to severe learning disability was present. Notably, although there was some evidence of behavioural and intellectual deterioration, there were no psychological or psychiatric difficulties in an emotionally stable woman who has good interpersonal relationships.

In the proband's residence, it was assumed that she was affected by Down's syndrome but the true karyotypic abnormality was discovered in 1976 when a blood sample was first submitted for chromosomal analysis.

\section{CHROMOSOME PREPARATIONS}

Metaphase spreads were prepared from phytohaemagglutinin (PHA) stimulated peripheral blood lymphocytes of the patient (for FISH) or healthy males (for CGH studies) using standard procedures of hypotonic treatment and methanol/acetic acid fixation (3:1 v/v).

CGH AND DIGITAL IMAGE ANALYSIS

Genomic DNA probe preparation, labelling procedures, hybridisation, and posthybridisation washings were carried out as described previously. ${ }^{16}$ Briefly, test and control DNA was extracted by proteinase $\mathrm{K}$ and RNase digestions. Control genomic DNA was prepared from blood of healthy females $(46, \mathrm{XX})$. Test (patient) human genomic DNA was directly labelled with FITC-12-dUTP (DuPont) and control DNA was labelled with Texas Red-5dUTP (DuPont) by standard nick translation reaction; DNase I concentration was adjusted to result in an average fragment size of 500-1000 bp. One microgram of fluorescein labelled test DNA, $1 \mu \mathrm{g}$ of Texas Red labelled reference, and $50 \mu \mathrm{g}$ of unlabelled human Cot-1 DNA (Gibco) were cohybridised to normal female metaphase spreads. Hybridisation was allowed to proceed for 48 hours at $37^{\circ} \mathrm{C}$ in a moist chamber. After posthybridisation washings, images for $\mathrm{CGH}$ analysis were acquired using an epifluorescence microscope (Axioplan, Zeiss, Germany) equipped with a cooled CCD camera (Photometrics) controlled by an image analysis system (Digital Scien- tific, Cambridge, UK). Green, red, and blue fluorescence images were captured from each high intensity, uniformly hybridised metaphase and were analysed as separate grey scale images. The image representing the blue DAPI counterstain was inverted and used for chromosome identification based on its coarse banding pattern. The mean of the individual ratio profiles of 20 metaphase spreads was calculated. For normalisation of the ratio profiles, the model value of the green to red ratio for the entire metaphase was set to 1.0. Finally, the individual ratio profiles were displayed next to the chromosome diagrams.

FLUORESCENCE IN SITU HYBRIDISATION

In an earlier attempt to identify the supernumerary chromosome, FISH to metaphase chromosomes was carried out using a number of commercially available centromeric probes (chromosomes 4, 12, 20, 22, 13/21, and 1/5/19). After the CGH results were obtained, targeted FISH was carried out using whole chromosome 19 paint (Oncor), 19 centromeric probe, $19 p$ and $19 q$ arm paints, and telomere specific probes for $19 p$ and $19 q(L I)$, according to the standard FISH procedures.

\section{Results}

CONVENTIONAL CYTOGENETIC ANALYSIS (FIG 2A)

Cytogenetic studies by $G$ banding in 1976 showed the presence of two cell lines. Eighteen out of 25 cells showed a 46,XX karyotype while seven out of 25 (28\%) showed an additional marker chromosome of unknown origin (46,XX/47,XX, +marker). Chromosome analysis by $G$ banding in 1986 confirmed the finding of two cell lines. Twenty-one out of 25 cells examined showed an apparently normal female karyotype while the remaining four cells (16\%) showed a count of 47 including one additional small chromosome. This marker appeared to be a ring chromosome, approximately half the size of a chromosome 21 , which contained at least one $\mathrm{G}$ band positive area (fig 2A). $\mathrm{C}$ banding showed one centromere to be present, and Ag NOR staining was negative with respect to the ring chromosome. No other members of the family underwent cytogenetic investigation.

\section{FIRST SERIES OF FISH STUDIES}

FISH was carried out using centromeric probes for chromosomes 4, 12, 20, 22, 13/21, and $1 / 5 / 19$ empirically. The studies failed to detect the origin of the extra chromosome and no further probes were investigated because difficulty was experienced in identifying the marker with certainty within the metaphases.

\section{CGH ANALYSIS (FIG 2B)}

CGH was carried out using a DNA sample from the patient. Analysis using a very restrictive threshold with a fluorescence ratio of 0.5 1.5 (which requires $100 \%$ abnormal cells to detect any gain or loss of the DNA material) failed to detect any abnormality. Progressively less restrictive thresholds were then used and, surprisingly, a threshold of 0.8-1.20 (which theoretically detects abnormal cells comprising 


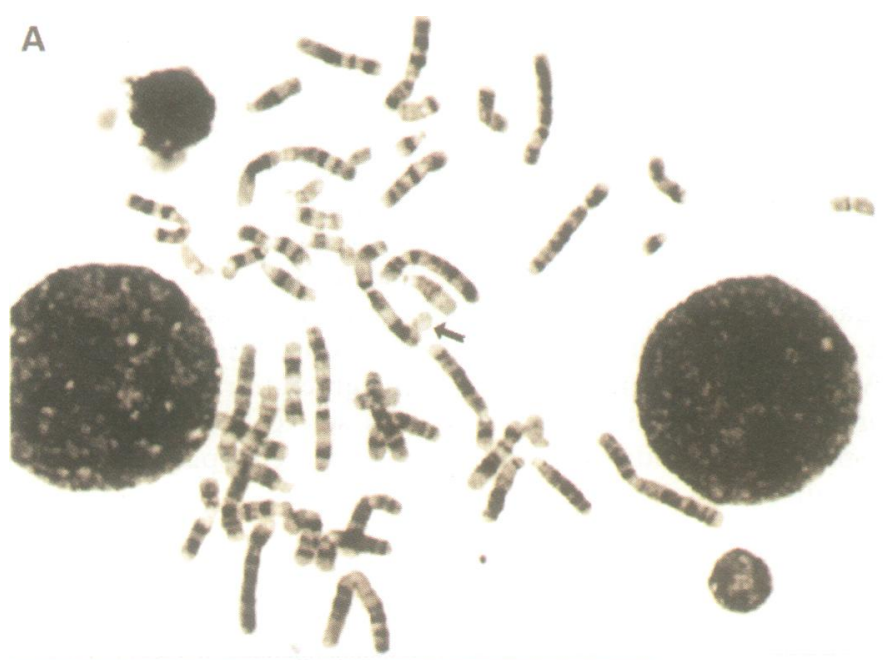

B
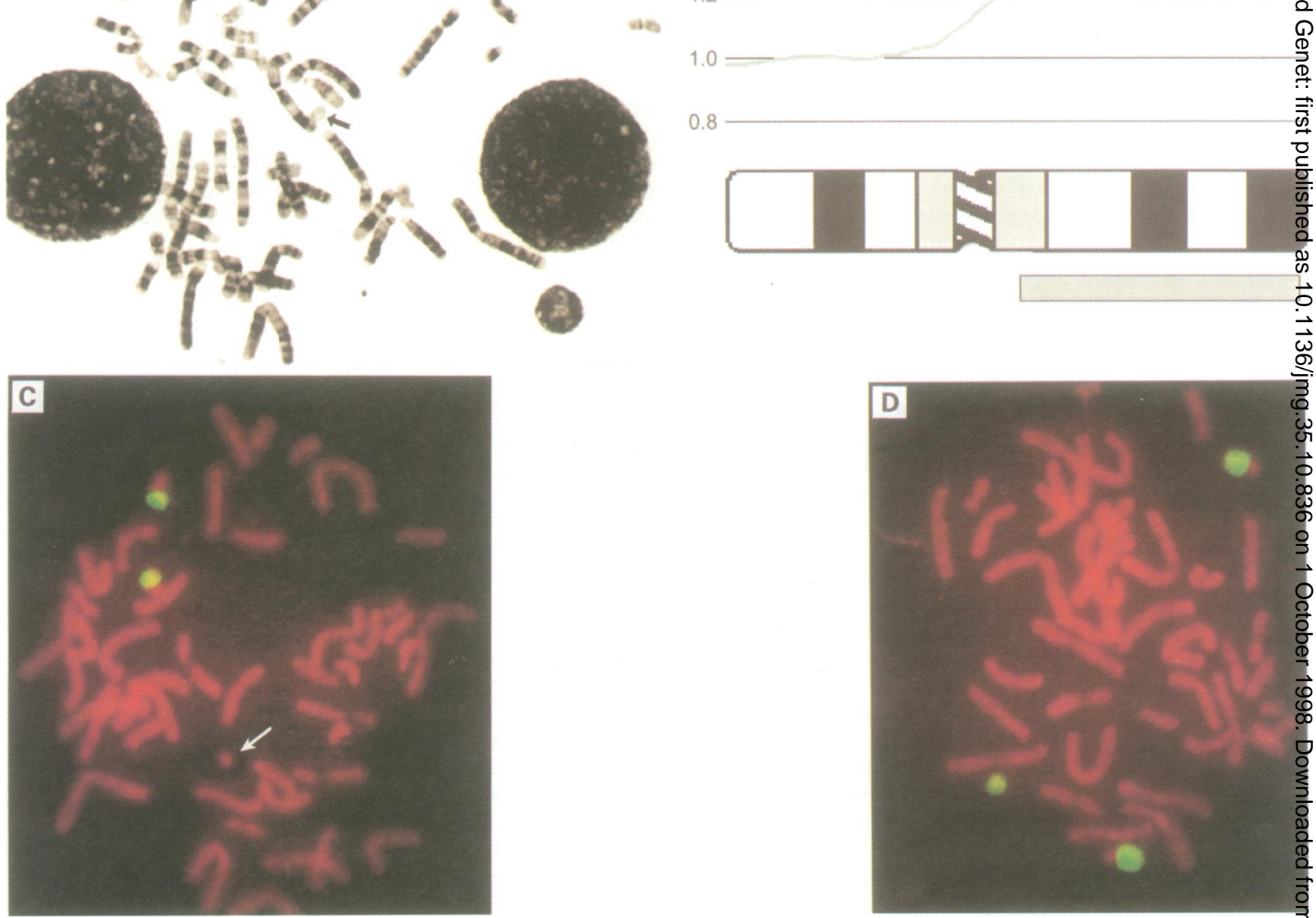

Figure 2 Conventional cytogenetic, $C G H$, and targeted FISH results. (A) $G$ banded metaphase spread of the patient showing a supernumerary ring chromosome (arrowed). (B) Standard ratio profile of chromosome 19 is shown above the chromosome idiogram. The horizontal lines represent the balance state of the chromosomal copy number (middle line, ratio 1.0) and the lower and upper thresholds applied to detect chromosomal losses and gains, respectively. These thresholds correspond to ratios of 0.8 and 1.20 , which are the theoretical values expected for a monosomy or trisomy present in $40 \%$ of diploid cells. (C,D) FISH to metaphase chromosome from the patient using chromosome 19p (C) and 19q arm specific painting probes (D).

$40 \%$ of the total) showed a gain in DNA material corresponding to $19 q$ (fig $2 B$ ). In $C G H$ the centromeric areas are strongly suppressed by unlabelled human Cot-1 DNA, so gain or loss of this area could not be interpreted reliably.

FISH CONFIRMATION OF THE CGH RESULTS (FIG 2C, D)

Further FISH studies using a whole chromosome 19 paint (WCP19, Oncor), 19p and 19q arm specific paints, and telomeric probes for $19 p$ and $19 q(\mathrm{LI})$ and 19 centromeric probe confirmed the CGH finding. The ring chromosome hybridised to the whole chromosome 19 and $19 q$ arm specific paints and chromosome 19 centromeric probe but not to $19 \mathrm{p}$ arm specific paint or $19 p$ or $19 q$ telomeric probes. It was thus concluded that the proband was trisomic for almost the entire long arm of chromosome 19 with only the telomeric region being absent from the ring.

\section{Discussion}

To date, only four cases with a supernumerary ring 19 have been reported. ${ }^{21718}$ Although there are 11 reported cases with partial $19 q$ trisomy, ${ }^{19-26}$ none is directly comparable to our case since in each instance non-homologous chromosomes were involved and the patterris of clinical abnormalities which resulted were quite variable.

Among the four cases with supernumerar ring 19 , one, a young infant with macrocepha. and developmental delay, is not directhy comparable as metaphase cells in that case contained dicentric marker chromosome 19 well as another marker chromosome, not 19 cs Among the remaining three cases, the first case $^{2}$ was a newborn infant aged 3 weeks with failure to thrive, hypotonia, and pneumonia with a marker chromosome 19 in $50 \%$ of the peripheral lymphocytes examined. The third case with supernumerary $r(19)^{18}$ had a ver small supernumerary $r(19)$ present in $46 \%$ of umbilical cord blood cells and estimated to be about $1 / 6$ the size of chromosome 21 . This was identified after amniocentesis was performeg for advanced maternal age. The normal mare infant weighed $3700 \mathrm{~g}$ at birth and he had not mal growth and development when evaluated aged 18 months. In another case, Quack et $a l^{17}$ reported a non-mosaic, supernumerary $r(19)$ present in a boy with somatic overgrowth and mental retardation. Unusually, this boy's 
mother carried the same ring 19 but had a balanced karyotype since one other chromosome 19 had an interstitial deletion of the long arm segment of chromosome 19 which formed the ring. Thus, the ring chromosome was shown to be derived from the deleted chromosome after the occurrence of two breaks, one in the centromere region, the other in band q13.2 in the long arm of chromosome 19 . There are few clinical resemblances between our elderly patient with ring 19 mosaicism and the non-mosaic child reported by Quack et $a l^{17}$; neither patient has any major congenital malformation, both have moderate mental handicap, but the striking difference is that our elderly patient has reduced stature and proportionately reduced head circumference, whereas the subject of the report of Quack et $a l^{17}$ has length, weight, and head circumference more than $3 \mathrm{SD}$ above the mean. Quack et $a l^{17}$ estimated that the IQ of their proband was 69 and reported particular difficulties with language, which is reminiscent of the findings in our patient whose psychological evaluations showed no evidence of a behavioural phenotype and only some evidence of age related gradual decline in intellect. In summary, in three cases non-specific moderate to mild mental retardation or developmental delay has been caused by mosaicism for an abnormal, supernumerary chromosome 19.

So far the approach to determining the origin of cytogenetically unidentifiable chromosomes has involved stepwise FISH using forward painting methods with alpha satellite, single copy, or whole chromosome painting probes, or reverse chromosome painting procedures with either flow sorting or microdissection of the particular chromosome. ${ }^{3-10}$ Microdissection followed by DOP-PCR amplification of the dissected DNA is increasingly being used and its modified approaches are reported to be so efficient that just one dissected DNA fragment is sufficient for a successful analysis. ${ }^{9}$ However, not all diagnostic cytogenetics laboratories have easy access to microdissection technology. Here we present an alternative approach which is not dependent on patient metaphase and is a genome wide screening technique enabling the result to be achieved in just one experiment. Many image analysis systems now in use in cytogenetic laboratories include software for $\mathrm{CGH}$, and results of analysis are available in three working days, which is comparable to the time required for microdissection. Where further confirmation by FISH is necessary one more day is required.

An advantage of $\mathrm{CGH}$ over microdissection is that, as shown here, it can provide an estimate of the proportion of cells containing the ring chromosome in the tissue studied. This information is laborious to obtain by chromosome analysis or interphase FISH. The minimum percentage of abnormal cells that can be detected by $\mathrm{CGH}$ has not yet been defined, but based on this report and our unpublished data, as little as $15 \%$ mosaicism in cultured metaphase cells could be detected. Theoretically, using a medium restrictive fluo- rescence ratio of $0.75-1.25$, there should be at least $50 \%$ abnormal cells present for the chromosome imbalance to be detected. ${ }^{27-29}$ In the present case, fluorescence ratio $0.8-1.20$ was the most restrictive threshold at which the abnormality was detected, which leads to an estimation of the percentage of abnormal cells in uncultured blood of at least $40 \%$. This proportion differs from the result of cytogenetic analysis in cultured cells which is based on a small sample of metaphases obtained from PHA stimulated $\mathrm{T}$ lymphocytes, and which showed not more than $28 \%$ abnormal cells. We currently use $\mathrm{CGH}$ to determine the origin of cytogenetically unidentifiable chromosomes and so far have obtained successful results in 10 cases with different sizes and percentages of ring and marker chromosomes (unpublished data). However, since in $\mathrm{CGH}$ the centromeric regions are suppressed, the method would not be applicable to ring or marker chromosomes composed almost entirely of centromeric heterochromatin.

The classical explanation for formation of a centric ring involves breaks in both arms of the chromosome with subsequent fusion of the proximal ends to form a ring and loss of the material distal to the breakpoints. More recently the possibility of $U$ type fusions or a $U$ type fusion in combination with transverse misdivision of the centromere has been put forward as a mechanism for the generation of small ring chromosomes. ${ }^{30}$ In the case described here, no short arm material could be seen in the ring chromosome either by $\mathrm{CGH}$ or by FISH using short arm paint, suggesting a breakpoint at or immediately adjacent to the centromere. No more detailed studies have yet been undertaken to define this breakpoint more precisely. CGH studies indicate that virtually the entire long arm of chromosome 19 is present in the ring, although by FISH the telomeric region is shown not to be present. We have previously shown that partial trisomy or partial monosomy of as little as 4-5 Mb can be detected in telomeric regions by $\mathrm{CGH} .{ }^{16}$ The fact that CGH has not shown any variation in copy number of any part of $19 q$ suggests that the breakpoint is within $5 \mathrm{Mb}$ of the telomere, and that the loss of long arm material in the formation of the ring chromosome is beyond the resolution of $\mathrm{CGH}$. However, although we have precisely identified the identity of the ring chromosome, we are not able to distinguish the mechanism by which it arose.

The first series of FISH experiments using empirically selected centromeric probes including one for chromosome 19 failed to indicate the origin of microdissection. This may be explained in part by the low percentage of cells containing the ring and difficulties in identifying it with certainty in some mitoses. Also the alpha satellite probe hybridising to chromosomes 1,5 , and 19 was used in the study, since at that time it was the only available centromeric probe for chromosome 19. It is possible that a small change in stringency conditions caused some loss of signal for chromosome 19. 
As discussed above, when no cytogenetic or clinical clues are available, a multicolour or stepwise FISH approach with or without chromosome microdissection may be used to identify the origin of a marker chromosome. However, in investigating small marker chromosomes, many service laboratories at present carry out sequential experiments using FISH probes that are selected empirically. Our experience indicates that in FISH studies the empirical approach may not detect a marker that is present in a lower percentage of metaphases, even though it is detected subsequently in a targeted FISH study. Although this experience is unlikely to be a frequent occurrence, it is one that is important for clinical cytogenetic practice.

We thank colleagues in the cytogenetics laboratories at the Duncan Guthrie Institute for preparing and examining many conventional cytogenetic preparations from the patient. We also thank Jackie Squire and Keith Bonham for expert psychological evaluations. SRG received a scholarship from the Ministry of Health and Medical Education, Iran.

1 Buckton KE, Spowart G, Newton MS, Evans HJ. Forty-four probands with additional "marker" chromosome. Hum Genet 1985;69:353-70.

2 Crolla JA, Dennis NR, Jacobs PA. A non-isotopic in situ hybridisation study of the chromosomal origin of 15 supernumerary marker

3 Blennow E, Telenius H, Larson C, et al. Complete characterization of a large marker chromosome by reverse and forward chromosome painting. Hum Genet 1992;90: 371-4.

4 Carter N, Ferguson-Smith MA, Perryman M, et al. Reverse chromosome painting: a method for rapid analysis of aberrant chromosomes in clinical cytogenetics. $\mathscr{f}$ Med Gene 1992;29:299-307.

5 Magnani I, Sacchi N, Darfler M, Nisson P, Tornaghi R, Fuhrmann-Conti A. Identification of the chromosome 14 origin of a C-negative marker associated with a $14 \mathrm{q} 32$ deletion by chromosome painting. Clin Genet 1993;43:1805.

6 Lengauer C, Eckelt A, Weith A, et al. Painting of defined chromosomal regions by in situ supression hybridization of libraries from laser-microdissection chromosomes. $C y$ togenet Cell Genet 1991;56:27-30.

7 Deng H, Yoshura K, Dirks R, et al. Chromosome-bandspecific painting; chromosome in situ suppression hybridization using PCR products from a microdissected chromosome band as a probe pool. Hum Genet 1992;89:13-17.

8 Ohta T, Tohma T, Soejima H, et al. The origin of cytologically unidentifiable chromosome abnormalities: six cases ascertained by targeted chromosome-band painting. Hum Genet 1993;92:1-5.

9 MullerNavia J, Nebel A, Schleiermacher E. Complete and precise characterization of marker chromosomes by precise characterization of marker chromosomes by application of microdiss
10 De A, Coelho KEF, Egashira M, et al. Diagnosis of four chromosome abnormalities of unknown origin by chromochromosome abnormalities of unknown origin by chromopainting. Am $\mathcal{F}$ Med Genet 1996;63:468-71.

11 Kallioniemi A, Kallioniemi OP, Sudar D, et al. Comparative genomic hybridization for molecular cytogenetic analysis solid tumors. Science 1992;258:818-21.

12 Kallioniemi OP, Kallioniemi A, Piper J, et al. Optimizin comparative genomic hybridization for analysis of DNA sequence copy number changes in solid tumors. Gent Chrom Cancer 1994;10:231-43.

13 Du Manoir S, Speicher MR, Joos S, et al. Detection of complete and partial chromosome gains and losses comparative genomic in situ hybridization. Hum Gers 1993;90:590-610.

14 Bryndorf $\mathrm{T}$, Kirchhoff $\mathrm{M}$, Rose $\mathrm{H}$, et al. Comparatife genomic hybridization in clinical cytogenetics. $\mathrm{Am} \mathrm{f} \mathrm{Hum}$ Genet 1995;57:1211-20.

15 Erdel M, Duba HC, Verdorfer I, et al. Comparative genominc hybridization reveals a partial de novo trisomy 6q23-qter in an infant with congenital malformations: delineation of phenotype. Hum Genet 1997;99:596-601.

16 Ghaffari SR, Boyd E, Tolmie J, Crow YJ, Trainer A $\overrightarrow{\mathrm{H}}$ Connor JM. A new strategy for cryptic telomeric translocia tion screening in patients with idiopathic mental retardat tion screening in patients with idiop
tion. $₹$ Med Genet 1998;35:225-33.

17 Quack B, Van RN, Verschraegen Spae MR, Klein $\underset{T}{T}$ Interstitial deletion and ring chromosome derived from 19q. Proximal 19q trisomy phenotype. Ann Genet 1992; 241-4.

18 Michalski K, Rauer M, Williamson N, Perszyk A, Hoo J] Identification, counselling, and outcome of two cases prenatally diagnosed supernumerary small ring chromio prematally diagnosed supernumerary sma

19 Lange M, Alfi OS. Trisomy 19q. Ann Genet 1976;19:17-29

20 Schmid W. Trisomy for the distal part of the long arm \&f chromosome 19 in brother and sister. Hum Genet 1979;46: 263-70.

21 Pangalos A, Ghica M, Couturier J. Trisomy 19q associated with partial trisomy 22q in two members of the same fan ily. Analysis using the thymidine and RBA bandiof techniques. Proceedings of the 6th international Congress $\mathbb{D} f$ techniques. Proceedings of the 6th internati

22 Zonana J, Brown MG, Magenis RE. Distal 19q duplicatio Hum Genet 1982;60:267-70.

23 Rivas F, Garcia-Cruz D, Rivera H, Plascencia ML, Gonzaleg RM. 19q distal trisomy due to a de novo $(19 ; 22)$ (q13 p11) translocation. Ann Genet 1985;28:113-15.

24 Madokoro H, Ohdo S, Sonodo T, Kawaguchi K, Ohba $\overline{\mathbf{K}}$ Partial trisomy for $19 \mathrm{q}$ due to paternal $17 / 19$ reciprofin translocation. Am $₹$ Hum Genet 1988;33:61-5.

25 Boyd E, Grass FS, Park JC, Knutson K, Stevenson R Duplication of distal 19q: clinical report and review. Ang Med Genet 1992;42:326-30.

26 Valerio D, Lavorgna F, Scalona M, Conte A. A new case $\overrightarrow{\overrightarrow{E f}}$ partial trisomy $19 \mathrm{q}$ (q13.2 $\rightarrow$ qter) owing to an unusug maternal translocation. F Med Genet 1993;30:697-9.

27 Du Manoir S, Schrock E, Bentz M, et al. Quantitative ana sis of comparative genomic hybridization. Cytometry 1999; 19:27-41.

28 Piper J, Rutovitz D, Sudar D, et al. Computer image analy of comparative genomic hybridization. Cytometry 1995; 10-26.

29 Du Manoir S, Kallioniemi OP, Lichter P, et al. Hardwa and software requirements for quantitative analysis of comparative genomic hybridization. Cytometry 1995;19:4-9. O

30 Callen DF, Eyre HJ, Ringenbergs ML, Freemantle $G$ Woodroffe P, Haan EA. Chromosomal origin of small ring marker chromosomes in man: characterization by molec lar genetics. Am f Hum Genet 1991;48:769-82. 